pate that the technique will be extensively applied to such materials over the next few years.

D. T. Clark is in the Department of Chemistry, University of Durham.

\section{Nuclear giant dipole resonance}

from P. E. Hodgson

LONG ago it was found that the cross sections of many reactions of gamma rays with nuclei, in particular those in which a nucleon is ejected, show broad resonances at quite high energies, usually around $20 \mathrm{MeV}$ for light nuclei and falling to about $10 \mathrm{MeV}$ for heavy nuclei. It was soon suggested that these resonances are due to a special type of collective excitation in which the neutrons vibrate against the protons. The resonance was found to be E1 (electric dipole) and a macroscopic calculation based on the liquid drop model gave approximately the correct variation of resonance energy with atomic weight.

Subsequently, detailed microscopic models have been developed that interpret the resonance as due to the coherent superposition of all possible particle-hole excitations from one major shell to the next. This was able to account for the width and the energy of the giant dipole resonances in several light nuclei.

The macroscopic models in terms of neutron and proton fluids were also developed, and these have the advantage that they give results for a whole range of nuclei for which the microscopic calculations would be very complicated. The most detailed of the macroscopic models are first that of Goldhaber and Teller (Phys. Rev. 74, $1046 ; 1948)$, which assumes that the oscillations of the neutron and proton fluids each take place within a fixed boundary, and this predicts that the excitation energy varies as $33 \mathrm{~A}^{-1 / 6}$. $\mathrm{A}$ second model, due to Steinwedel and Jensen (Z. Naturforsch. 5A, 413; 1950) assumes that the neutron-proton

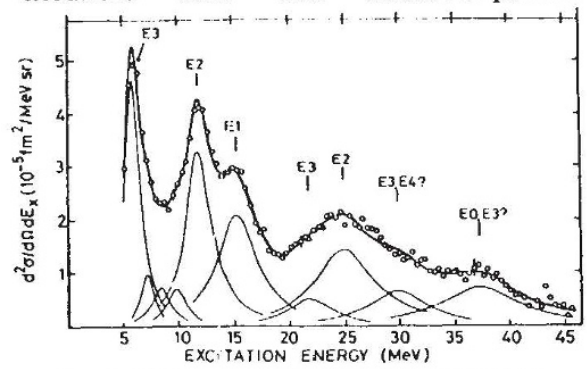

Fig. 1 Spectrum of $92.1 \mathrm{MeV}$ electrons inelastically scattered from ${ }^{140} \mathrm{Ce}$ at 90 , showing the peaks due to the electric resonances of the multipolarities indicated. This spectrum is analysed into its components. oscillation takes place within one fixed boundary, and this gives $80 \mathrm{~A}^{-1 / 3}$ for the energy dependence. More recently, Myers and Swiatecki (Phys. Rev. C15, 2032; 1977) have developed a more detailed theory based on their droplet model of the nucleus, and this gives an energy variation of $\mathrm{A}^{-0.23}$, which is nearer to the experimental results than the other models. The models also predict distinctly different transition charge densities.

To test these models, Pitthan and colleagues at the Naval Postgraduate School, Monterey, California have recently made some careful measurements of the inelastic scattering of electrons by ${ }^{140} \mathrm{Ce}$. (Phys. Rev. Lett. 41, $1276 ; 1978)$. This nucleus was chosen because of all the nuclei investigated it shows the most favourable separation between the various possible resonances. The spectrum of inelastically scattered 91.2 MeV electrons that they obtained is shown in Fig. 1, with the background already subtracted. A number of peaks are visible, corresponding to the electric resonances of various multipolarities. The spectrum is analysed into the contributions from the different multipolarities, and the one of interest for distinguishing between the different models is the dipole resonance at about $15.3 \mathrm{MeV}$. Similar measurements were made at a number of incident electron energies from 65 to $150 \mathrm{MeV}$.

The results for the dipole resonances at different electron energies were compared with the models of the excitation process by plotting the ratio of the experimental cross section to the Mott cross section at a particular angle as a function of the momentum transfer, and this is compared with predictions of the three theories in Fig. 2. It is clear that the MyersSwiatecki model agrees very well with the data, whereas the SteinwedelJensen model predicts cross sections that are too high.

The Goldhaber-Teller model predicts cross sections that are too low, but it is just possible that the shortfall could be attributed to the presence of an undetected monopole (breathing mode) resonance. This state, in which the nucleus oscillates radially, has been predicted theoretically, and some evidence for its presence has been found in previous experimental work (see for example News \& Views 271, 212 ; 1978). To study this possibility, the difference between the predictions of the Goldhaber-Teller model and the experimental results was compared with distorted wave calculations of the contribution to be expected from a monopole excitation. The overall dependence

P. E. Hodgson is a Lecturer in Nuclear Physics in the University of Oxford.

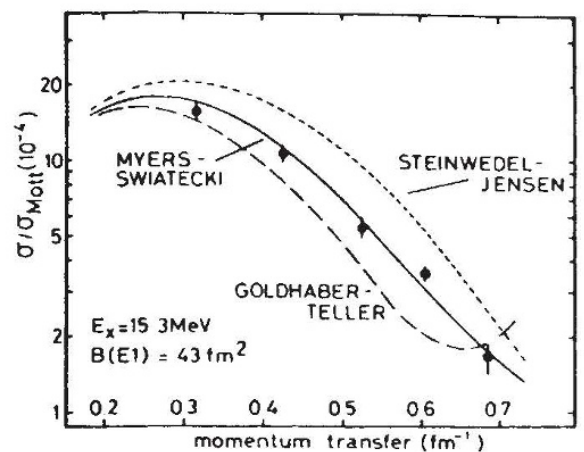

Fig. 2 Comparison between the experimental results of the electric dipole resonance at several values of the momentum transfer and the predictions of three theories of the excitation process.

on momentum transfer is found to be the same, and if it is indeed monopole it exhausts about $45 \%$ of the energyweighted sum rule. This interpretation is however doubtful partly because the variation with $\mathrm{A}$ is already in disagreement with the Goldhaber Teller model, and partly because if the monopole interpretation is correct we would expect to see all the energy-weighted sum. Thus at present the evidence favours the Myers-Swiatecki model for the giant dipole resonance. In addition, there is some discrepancy between the evidence in favour of the monopole state obtained from measurements of inelastic alpha-particle scattering and its absence in the more thoroughly understood inelastic electron scattering. Further measurements of both electron and hadron inelastic scattering are needed to resolve this discrepancy.

\section{Physics in archaeology}

from Robert Hedges

Traditionally, Archaeometry Symposia focus on the development and application of physical methods to archaeology, and there were no papers on environmental or biological issues, although two studies on the survival and extraction of organic residues were presented. Broad categories, often leading to parallel sessions, of dating, prospection, artefact analysis with the aims both of reconstructing ancient technologies, and determining the original point of manufacture, and mining and extractive metallurgy continued to provide the bulk of the research reports. *The 19th International Symposium on Archaeometry and Archaeological Pros 